\title{
Diurnal variation of vascular diameter and reactivity in healthy young men
}

\author{
P.F.D. Bau',2, C.H.D. Baư ${ }^{3}$, A.A. Naujorks², G.A. Rosito ${ }^{4}$ and F.D. Fuchs ${ }^{5}$ \\ ${ }^{1}$ Departamento de Clínica Médica, Centro de Ciências da Saúde, Universidade Federal de Santa Maria, \\ Santa Maria, RS, Brasil \\ ${ }^{2}$ Instituto Cardiovascular, ICARDIO, Santa Maria, RS, Brasil \\ ${ }^{3}$ Departamento de Genética, Instituto de Biociências, Universidade Federal do Rio Grande do Sul, \\ Porto Alegre, RS, Brasil \\ ${ }^{4}$ Departamento de Medicina Preventiva, Fundação Faculdade Federal de Ciências Médicas de Porto \\ Alegre, Porto Alegre, RS, Brasil \\ ${ }^{5}$ Serviço de Cardiologia, Hospital de Clínicas de Porto Alegre, Porto Alegre, RS, Brasil
}

Correspondence to: P.F.D. Bau, Rua Appel, 655/903, 97015-030 Santa Maria, RS, Brasil

Fax: +55-3025-1212. E-mail: paulobau@gmail.com

\begin{abstract}
The higher incidence of cardiovascular events in the morning is accompanied by an increased vascular tone. However, there are few published studies designed to evaluate the diurnal variation of vascular and endothelial parameters in healthy subjects. In the present investigation, we evaluated the diurnal variation in brachial artery diameter (BAD), flow-mediated dilation (FMD) and endothelium-independent dilation (NFMD) in a homogeneous sample of healthy non-smoker young men. Fifty subjects aged $20.8 \pm 0.3$ years (range: 18 to 25 years) were investigated by brachial artery ultrasound. Exclusion criteria were female gender and evidence of clinically significant health problems, including obesity. Volunteers were asked to rest and avoid fat meals as well as alcoholic beverages $48 \mathrm{~h}$ before and until completion of the evaluations. BAD, FMD and NFMD were measured at 7 am, $5 \mathrm{pm}$, and $10 \mathrm{pm}$ and tested by repeated measures ANOVA. BAD was smaller at $7 \mathrm{am}$ (mean $\pm \mathrm{SEM}, 3.8 \pm 0.1 \mathrm{~mm}$ ) in comparison with $5 \mathrm{pm}(3.9 \pm 0.1)$ and $10 \mathrm{pm}(4.0 \pm 0.1 \mathrm{~mm} ; \mathrm{P}<0.001)$. FMD values did not change significantly during the day, while NFMD increased more at $7 \mathrm{am}(18.5 \pm 1.1 \%)$, when compared to $15.5 \pm 0.9 \%$ at $10 \mathrm{pm}$ and $15.5 \pm 0.9 \%$ at $5 \mathrm{pm}(\mathrm{P}=0.04)$. The physiological state of vasoconstriction after awakening, with preserved capability to dilate in the morning, should be considered to be part of the healthy cardiovascular adaptation before considering later life risk factors and endothelial dysfunction.
\end{abstract}

Key words: Cardiovascular risk factor; Circadian rhythm; Brachial artery; Vasoconstriction; Vascular biology

Received June 15, 2007. Accepted March 4, 2008

\section{Introduction}

Cardiovascular events such as sudden death, stroke and acute coronary syndromes have a higher incidence in the period surrounding awakening (1-3). This vulnerable period has been ascribed to cardiocirculatory (3) and hemostatic (4) adaptations typical of this period of day. Changes in these adaptations have been implicated both in the genesis of atherosclerosis (5) and in the higher incidence of cardiovascular events during the morning period (6).

A plethysmographic study of blood flow and vascular resistance in the forearm indicated that the circadian rhythm of vascular tone is related to increased alpha-sympathetic vasoconstrictor activity in the morning (7). This study was followed by others which analyzed the relationships between vascular and endothelial physiology and the increased frequency of cardiovascular events in the morning. Some of these were Doppler ultrasound studies carried out to determine brachial artery diameter (BAD) and its change after a stimulus, allowing inferences about the endothelial function (8). These measures are the endothelium-dependent flow-mediated dilation (FMD) and endothelium-independent nitroglycerin-mediated dilation (NFMD). Part of these studies identified circadian variations and blunted responses during the morning period in 
individuals with $(9,10)$ or without $(11)$ a higher risk of developing cardiovascular events. These findings were not reproduced by others $(4,12,13)$. The inconsistency of findings related to the day-night and time of day variation in BAD and FMD could be secondary to the low statistical power of some studies and to the co-existence of different conditions that may influence vascular function, such as risk factors for vascular disease $(12,14)$, use of medications (14), gender $(10,14,15)$, age $(14)$, phase of the menstrual cycle $(10,15)$, nutrition (14), and environmental temperature (10). Therefore, there is a lack of controlled studies specifically designed to evaluate the circadian variation in these parameters by Doppler ultrasound.

In order to determine which morning variations are physiological and present before the appearance of risk factors at an older age, we addressed the influence of time of day on BAD, FMD and NFMD in a homogeneous sample of healthy young men. If these young subjects present either lower FMD or vasoconstriction in the morning, it can be assumed that such characteristics are not the consequence of other risk factors.

\section{Subjects, Material and Methods}

The Ethics Committee of our institution approved the study and all participants agreed to participate after reading a detailed informed consent form. The individuals evaluated were 50 male subjects of the control group of a study on the effects of ethanol on cardiovascular parameters (16).

All volunteers were evaluated at the echocardiography laboratory of the Charity Hospital of Santa Maria. They were asked to abstain from alcohol and other psychoactive substances $48 \mathrm{~h}$ before the study. Volunteers arrived at the laboratory at $4 \mathrm{pm}$ and were evaluated by clinical history and physical examination, including the measurement of weight, height, and blood pressure. Afterwards, they rested for $10 \mathrm{~min}$ before beginning the first evaluation, done at $5 \mathrm{pm}$.

$B A D$ and flow velocity measures were obtained according to the guidelines for the ultrasound assessment of endothelial-dependent flow-mediated vasodilation of the brachial artery (8) using an ultrasound instrument with a $10-\mathrm{MHz}$ linear transducer (Aspen, Acuson Computed Sonography, USA). For each volunteer, optimized images of the brachial artery were obtained above the antecubital fossa of the right arm. This position was marked and all subsequent images were obtained at the same site. Vascular measurements were performed in the supine position in a quiet, temperature-controlled room of the echocardiography laboratory, with continuous electrocardiographic monitoring.
Initially, longitudinal two-dimensional images were acquired. Arterial flow velocity measurements were acquired using a pulsed Doppler signal at $60^{\circ}$ to the vessel with the range gate $(1.5 \mathrm{~mm})$ in the center of the artery. After the baseline recording of BAD, a cuff was inflated in the forearm to $220 \mathrm{mmHg}$ for $5 \mathrm{~min}$, leading to arterial occlusion. During the procedure, the transducer position was carefully maintained. Following the rapid deflation of the cuff, pulsed Doppler signals were recorded for $15 \mathrm{~s}$, and longitudinal images were assessed after $90 \mathrm{~s}$. Thereafter, a 10-min period was allowed for recovery of the vessel. Longitudinal images were then obtained before and $4 \mathrm{~min}$ after the administration of $400 \mu \mathrm{g}$ of sublingual nitroglycerin spray. Measurements were performed in three consecutive cardiac cycles at the peak of the $R$ wave of the electrocardiogram. The measures were later averaged. All images were recorded on VHS for later analysis. Intraobserver variability, i.e., the mean difference between the measurements for each individual, was $<3 \%$ for all measurements.

The measurements were repeated at $10 \mathrm{pm}$ and $7 \mathrm{am}$. A standard low-fat meal was served at $8 \mathrm{pm}$. After the $10-$ pm evaluation, volunteers received a snack and were allowed to sleep in the laboratory until 6:30 am. Breakfast was served after the 7-am evaluation. Therefore, in the 7am and 5-pm evaluations, patients had been fasting for more than $4 \mathrm{~h}$, while the 10-pm evaluation was performed $2 \mathrm{~h}$ after a light, low-fat meal.

Data are reported as means \pm SEM. The time effect on systolic blood pressure, diastolic blood pressure, heart rate, $B A D, F M D$, and NFMD variation in the three measurements was evaluated by repeated measures ANOVA. Between-time post hoc analyses were performed with Bonferroni adjustment for repeated measures using the SPSS software (Version 12.0; SPSS Inc., USA).

\section{Results}

\section{Sample characteristics}

The sample consisted of 50 healthy, volunteer, nonsmoker men aged $18-25$ years $(20.8 \pm 0.3$ years $)$, with no history of cardiovascular disease or use of medication. Their average body mass index was $23.1 \pm 0.3 \mathrm{~kg} / \mathrm{m}^{2}$ (range: 18.5 to 28.7 ). Table 1 shows that both heart rate and blood pressure were higher at $5 \mathrm{pm}$.

\section{Vascular measurements}

BAD did not differ at $5 \mathrm{pm}$ and $10 \mathrm{pm}(3.9 \pm 0.1$ and 4.0 $\pm 0.1 \mathrm{~mm}$, respectively), but was significantly smaller at 7 am (3.8 $\pm 0.1 \mathrm{~mm}$, Figure 1).

Flow-mediated dilation was constant through the day 
and night, being $5.3 \pm 0.5 \%$ at $7 \mathrm{am}, 5.1 \pm 0.6 \%$ at $5 \mathrm{pm}$, and $5.1 \pm 0.6 \%$ at $10 \mathrm{pm}$ (repeated measures ANOVA).

A significant increase in NFMD was observed at 7 am $(18.5 \pm 1.1 \%)$, compared to $15.5 \pm 0.9 \%$ at $5 \mathrm{pm}$ and 15.5 $\pm 0.9 \%$ at $10 \mathrm{pm}$ on the previous day (Figure 2 ).

\section{Discussion}

The present study reports the diurnal variation of vascular diameter, evaluated by vascular ultrasound, of healthy young males. The smaller diameter in the morning accompanied by a normal capability to dilate represents an important feature of the physiological adaptation of these parameters after awakening. When individuals get older and have other risk factors related to endothelial dysfunction, even relatively minor degrees of vasoconstriction in the morning may critically reduce blood flow (7).

The cardiovascular adaptation in the morning, characterized by an increase in vascular tone, alpha-sympathetic vasoconstrictor activity, and catecholamine levels, has been reported for other age groups $(7,13)$. As far as we know, our study is the first Doppler ultrasound study to describe smaller BAD in the morning period, contrasting with findings described by others $(6,11)$. This lower BAD is consistent with the increase in peripheral resistance expected at this time of day. The fact that a lower heart rate accompanied the morning vasoconstriction could be explained by a circadian pattern of cardiac output and total peripheral resistance originating from the day-night pattern in physical activity (17). During the nighttime, blood flow to the skeletal muscles is decreased through local autoregulation, which increases total peripheral resistance and decreases cardiac output compared with daytime (17). Therefore, our results are in accordance with the overall cardiovascular adaptation expected after awakening and assuming the upright position. The interpretation that arteries were constricted at that time, maintaining their physiological capability to dilate, was additionally confirmed by the higher NFMD during the morning period. In fact, previous studies have shown that NFMD is inversely correlated with arterial diameter (18).

Although our protocol included FMD measurements, the results should be interpreted with caution since recent studies (19-21) have suggested the need to correct FMD for the stimulus (shear stress). These studies showed that several risk factors for cardiovascular disease actually impact the shear stress itself, instead of endothelial function. A major issue is that FMD does not always reflect nitric oxide activity (21). Unfortunately, the evaluation of shear stress was not thoroughly addressed in the guidelines followed here (8). This scenario suggests that the clinical significance of time of day variation in FMD reported in previous studies $(6,9-11)$ is controversial.

Considering the time of day differences in BAD, the conclusions associated with endothelial function are difficult to interpret. There is a clear need for more studies specifically designed to evaluate the diurnal and circadian variation in these parameters before definitive conclusions about the morning surge in events can be reached. Most

Table 1. Blood pressure and heart rate at the different times of vascular evaluation.

\begin{tabular}{lrrrc}
\hline & \multicolumn{1}{c}{$7 \mathrm{am}$} & \multicolumn{1}{c}{$5 \mathrm{pm}$} & \multicolumn{1}{c}{$10 \mathrm{pm}$} & $\mathrm{P}$ \\
\hline HR (bpm) & $62.0 \pm 1.1$ & $72.2 \pm 1.5$ & $68.8 \pm 1.4$ & $<0.001$ \\
SBP (mmHg) & $111.9 \pm 1.3$ & $116.3 \pm 1.3$ & $113.6 \pm 1.3$ & 0.02 \\
DBP (mmHg) & $67.5 \pm 1.2$ & $70.9 \pm 1.3$ & $65.8 \pm 1.2$ & 0.008
\end{tabular}

Data are reported as mean $\pm \mathrm{SEM}$. $\mathrm{HR}=$ heart rate; $\mathrm{SBP}=$ systolic blood pressure; DBP = diastolic blood pressure. In the post hoc comparisons (with Bonferroni adjustment), HR, SBP, and DBP were higher at $5 \mathrm{pm}$ (repeated measures ANOVA).

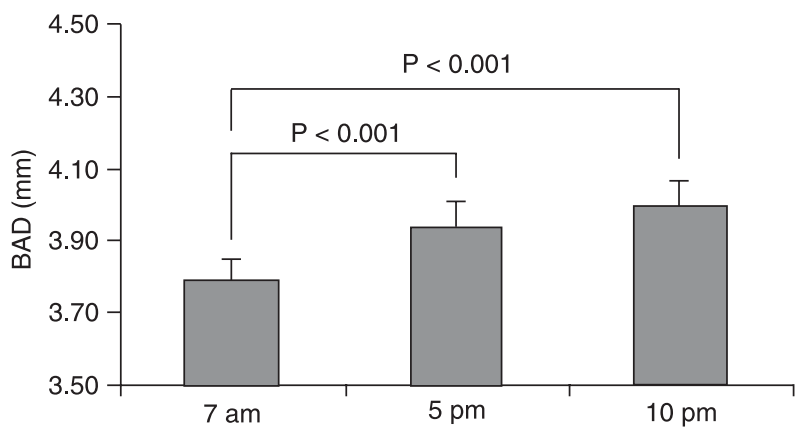

Figure 1. Brachial artery diameter (BAD) at different times of day. Data are reported as means \pm SEM. $P<0.001$ (repeated measures ANOVA). Significant post hoc differences are presented.

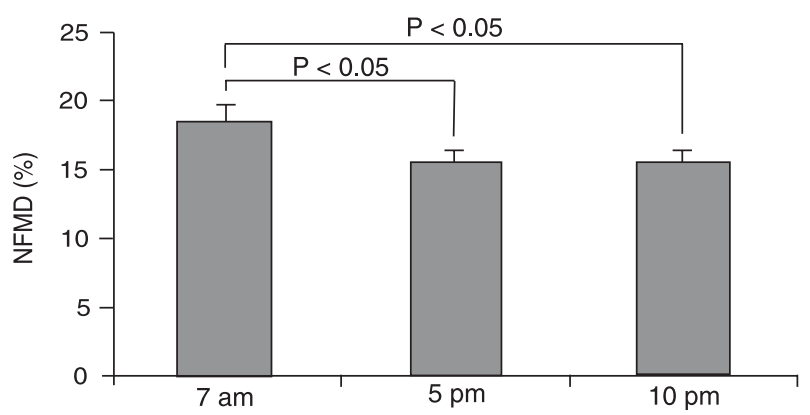

Figure 2. Endothelium-independent dilation (NFMD) measured at different times of day. Data are reported as means \pm SEM. $P=$ 0.04 (repeated measures ANOVA). A significant post hoc difference is presented. 
importantly, the diurnal variation in BAD confounds the results of endothelial function in the early morning. A weakness of the present study was the lack of a more detailed clinical assessment (such as laboratory tests). The young volunteers were considered to be healthy based only on clinical information (anamnesis and physical examination). Another limitation pertains to gender. The fluctuations in estrogen concentrations during the menstrual cycle are reflected in changes in levels of endothelial function markers (15). The present study should be re- peated in females in order to determine if there are gender similarities in the diurnal variation in vascular diameter and reactivity.

We demonstrated that, after awakening, young males present a physiological state of vasoconstriction, not accompanied by changes in endothelial function. Changes in this pattern throughout the life span should be taken into account in future studies of the morning peak in cardiovascular events.

\section{References}

1. Muller JE, Stone PH, Turi ZG, Rutherford JD, Czeisler CA, Parker $\mathrm{C}$, et al. Circadian variation in the frequency of onset of acute myocardial infarction. N Engl J Med 1985; 313: 1315-1322.

2. Cannon $\mathrm{CP}$, McCabe $\mathrm{CH}$, Stone $\mathrm{PH}$, Schactman $\mathrm{M}$, Thompson $\mathrm{B}$, Theroux $\mathrm{P}$, et al. Circadian variation in the onset of unstable angina and non-Q-wave acute myocardial infarction (the TIMI III Registry and TIMI IIIB). Am J Cardiol 1997; 79: 253-258.

3. Hu K, Ivanov PC, Hilton MF, Chen Z, Ayers RT, Stanley HE, et al. Endogenous circadian rhythm in an index of cardiac vulnerability independent of changes in behavior. Proc Natl Acad Sci U S A 2004; 101: 18223-18227.

4. Shaw JA, Chin-Dusting JP, Kingwell BA, Dart AM. Diurnal variation in endothelium-dependent vasodilatation is not apparent in coronary artery disease. Circulation 2001; 103: 806-812.

5. Schachinger $\mathrm{V}$, Britten MB, Zeiher AM. Prognostic impact of coronary vasodilator dysfunction on adverse long-term outcome of coronary heart disease. Circulation 2000; 101: 1899-1906.

6. Otto ME, Svatikova A, Barretto RB, Santos S, Hoffmann M, Khandheria B, et al. Early morning attenuation of endothelial function in healthy humans. Circulation 2004; 109: 25072510.

7. Panza JA, Epstein SE, Quyyumi AA. Circadian variation in vascular tone and its relation to alpha-sympathetic vasoconstrictor activity. N Engl J Med 1991; 325: 986-990.

8. Corretti MC, Anderson TJ, Benjamin EJ, Celermajer D, Charbonneau F, Creager MA, et al. Guidelines for the ultrasound assessment of endothelial-dependent flow-mediated vasodilation of the brachial artery: a report of the International Brachial Artery Reactivity Task Force. J Am Coll Cardiol 2002; 39: 257-265.

9. Elherik K, Khan F, McLaren M, Kennedy G, Belch JJ. Circadian variation in vascular tone and endothelial cell function in normal males. Clin Sci 2002; 102: 547-552.

10. Gaenzer H, Sturm W, Kirchmair R, Neumayr G, Ritsch A, Patsch J. Circadian variation of endothelium-dependent vasodilatation of the brachial artery as a confounding factor in the evaluation of endothelial function. Atherosclerosis 2000; 149: 227-228.
11. Etsuda $H$, Takase $B$, Uehata $A$, Kusano $H$, Hamabe $A$, Kuhara $\mathrm{R}$, et al. Morning attenuation of endothelium-dependent, flow-mediated dilation in healthy young men: possible connection to morning peak of cardiac events? Clin Cardiol 1999; 22: 417-421.

12. Kawano H, Motoyama T, Yasue H, Hirai N, Waly HM, Kugiyama K, et al. Endothelial function fluctuates with diurnal variation in the frequency of ischemic episodes in patients with variant angina. J Am Coll Cardiol 2002; 40: 266270.

13. Ringqvist A, Caidahl K, Petersson AS, Wennmalm A. Diurnal variation of flow-mediated vasodilation in healthy premenopausal women. Am J Physiol Heart Circ Physiol 2000; 279: $\mathrm{H} 2720-\mathrm{H} 2725$.

14. Vapaatalo $\mathrm{H}$, Mervaala $\mathrm{E}$. Clinically important factors influencing endothelial function. Med Sci Monit 2001; 7: 10751085.

15. Walters J, Skene D, Hampton SM, Ferns GA. Biological rhythms, endothelial health and cardiovascular disease. Med Sci Monit 2003; 9: RA1-RA8.

16. Bau PF, Bau $\mathrm{CH}$, Naujorks AA, Rosito GA. Early and late effects of alcohol ingestion on blood pressure and endothelial function. Alcohol 2005; 37: 53-58.

17. Veerman DP, Imholz BP, Wieling $\mathrm{W}$, Wesseling $\mathrm{KH}$, van Montfrans GA. Circadian profile of systemic hemodynamics. Hypertension 1995; 26: 55-59.

18. Schroeder S, Enderle MD, Baumbach A, Ossen R, Herdeg $C$, Kuettner $A$, et al. Influence of vessel size, age and body mass index on the flow-mediated dilatation (FMD\%) of the brachial artery. Int J Cardiol 2000; 76: 219-225.

19. Mitchell GF, Vita JA, Larson MG, Parise H, Keyes MJ, Warner $\mathrm{E}$, et al. Cross-sectional relations of peripheral microvascular function, cardiovascular disease risk factors, and aortic stiffness: the Framingham Heart Study. Circulation 2005; 112: 3722-3728.

20. Deanfield JE, Halcox JP, Rabelink TJ. Endothelial function and dysfunction: testing and clinical relevance. Circulation 2007; 115: 1285-1295.

21. Pyke KE, Tschakovsky ME. The relationship between shear stress and flow-mediated dilatation: implications for the assessment of endothelial function. J Physiol 2005; 568: 357369. 